\title{
Experimental Evaluation of the Wake Characteristics of Cross Flow Turbine Arrays
}

\author{
Stephanie Ordonez-Sanchez ${ }^{\mathrm{a}, *}$, Duncan Sutherland ${ }^{\mathrm{b}}$, Grégory S. Payne ${ }^{\mathrm{b}}$, Tom Bruce ${ }^{\mathrm{b}}$, Mulualem \\ Gebreslassie $^{\mathrm{d}}$, Michael R. Belmont ${ }^{\mathrm{c}}$, Ian Moon ${ }^{\mathrm{c}}$ \\ ${ }^{a}$ Energy Systems Research Unit, Department of Mechanical \& Aerospace Engineering, University of Strathclyde, James Weir \\ Building, Montrose Street, Glasgow G1 1 XJ, United Kingdom \\ ${ }^{b}$ Institute for Energy Systems, School of Engineering, University of Edinburgh, King's Buildings, Edinburgh, EH9 3DW, \\ United Kingdom \\ ${ }^{c}$ College of Engineering, Mathematics and Physical Sciences, University of Exeter, North Park Road, Exeter EX4 4 QF, \\ United Kingdom \\ ${ }^{d}$ School of Mechanical and Industrial Engineering, EiT-M, Mekelle University, P.O Box 231, Mekelle, Tigray, Ethiopia
}

\begin{abstract}
One key factor in the exploitation of tidal energy is the study of interactions of turbines when working in tidal turbine farms. The Momentum Reversal and Lift (MRL) turbine is a novel cross flow turbine. The three blades rotate around a common central horizontal axis which is parallel to their own axis and perpendicular to the flow. The novelty of the MRL turbine is that it relies on the combination of both lift and momentum reversal (drag) for energy extraction. Scaled MRL turbine models of $0.164 \mathrm{~m}$ in diameter were used to characterise the flow in three different tidal array settings. Detailed maps of axial velocity profiles and velocity deficits downstream of the turbine are presented, enabling the visualisation of characteristic flow patterns. The results show that the MRL generates lower velocity deficits and turbulence intensities in the near wake than those associated with horizontal axis turbines. The downstream wake was not completely symmetrical which was related to the geometry of the device but also due to the flow developed in the flume. Amongst the three array configurations studied, a fence of turbines with the lowest separation provided the highest power output.
\end{abstract}

Keywords: Marine Renewables, Tidal Stream Energy, Arrays, Scale Testing, Physical Modelling, Wake Interactions.

\section{Nomenclature}

MRL $=$ Momentum Reversal Lift Turbines $\mathrm{BSR}=$ Blade Speed Ratio

\footnotetext{
*s.ordonez@strath.ac.uk

$U R L$ :
}

www.strath.ac.uk/esru/research/hydrowavetidalpower/ (Stephanie Ordonez-Sanchez), www.eng.ed.ac.uk/research/institutes/ies (Duncan Sutherland), www.eng.ed.ac.uk/research/institutes/ies (Grégory S. Payne),

www.eng.ed.ac.uk/research/institutes/ies (Tom Bruce),

www . emps.exeter.ac.uk/engineering/research/cws (Mulualem Gebreslassie),

www.emps.exeter.ac.uk/engineering/research/cws (Michael R. Belmont), www.emps. exeter.ac.uk/engineering/research/cws (Ian Moon )

Preprint submitted to Ocean Engineering
HATT $=$ Horizontal Axis Tidal Turbine

$\mathrm{I}=$ Turbulence Intensity.

\section{Introduction}

The tidal energy industry is advancing to a stage were commercial deployments are expanding to arrays of multiple devices. The interaction of flow through and around tidal turbines is crucial to understand the energy shadowing of an array and how this influences energy extraction by the individual devices. Consequently, researchers have focused on quantifying the flow features around tidal turbines using numerical modelling and experimental work.

Studies done by $[1,2,3,4,5]$ have utilised actuator disc approximation methods to investigate the flow characteristics of individual turbines and

June 13, 2017 
arrays. The actuator disc method is a highly simplified momentum absorption zone and has no capability of resolving the flow around each blade, only reducing the momentum of the fluid as it passes through the disc [2]. A study done by Harrison et. al. [1] also indicated that the vortex shedding from the edge of the disc is not similar to rotating blades and lacks swirl producing flow. Using the MRL turbine, work has focused on expanding the actuator disk theory to an immersed body forced (IBF) model with directional effects. This work has covered a single device and arrays $[6,7,8,9]$.

Experimental studies have been carried out to investigate the wake interaction in a tidal stream farm experimentally. Initial research on arrays has included porous disks which can remove energy from the flow and have the potential to measure thrust. Myers and Bahaj [10] compare arrays of a series of porous disks of $1 / 20$ th with multiple-device arrangements to $1 / 20$ th horizontal axis turbines. The objective was to compare the near and far wake of both models. They found that the near wake is highly turbulent and this is difficult to model with porous discs while the comparative results show similarities in the far wake region for both models. However they do not impart rotational effects on the flow. Mycek et al. [11] undertook a study of two interacting dynamic (rotating) Horizontal Axis Tidal Tubines (HATTs) spaced downstream. The experimental work was done with two different settings of turbulence intensity, showing that as the turbulence intensity is increased, the performance of the downstream turbine increases with an associated reduction of wake effects. Stallard et al. [12] also carried out an experimental analysis of an array of a number of dynamic HATTs, with results focused on downstream and lateral wake recovery. More recently, Jeffcoate et al. [13] quantified the performance of two turbines when interacting at different spacings, their investigation was completely focused on power and thrust measurements and no wake characterisation was included.

In the majority of the literature the experimental analysis of arrays are highly focused on horizontal axis turbines and there is still uncertainty as to the similarities and differences in wake effects between other turbine designs. Moreover, the effects of wake expansion associated with the site depth have not been studied for vertical axis turbines which might be of interest for large turbine designs in shallow water depths. These studies are particularly important in turbine arrays where high blockage ratios will cause significant bypass flows which may cause downstream flows to approach critical condition.

There are still significant steps to be taken in understanding the wake of dynamic turbine models including swirl and associated mixing. These effects get more complicated when the flow in the wakes of these devices interact. This work, along with the related CFD [14], seeks to improve characterisation of the flow through arrays of dynamic turbine scalemodels. This work utilises the Momentum Reversal lift Turbine, which is designed for high blockage conditions such as estuaries. The first stage of the work presented in this paper focuses on the study of a single MRL turbine by characterising its efficiency and flow field. This is then expanded to multiple devices in three array configurations using 3-4 devices. The main purpose of this paper is to gain a deeper understanding of flow effects due to the MRL turbine when working as a single device and within simple array configurations.

\section{Methodology}

\subsection{The MRL Turbine}

The Momentum Reversal and Lift (MRL) turbine is a cross flow turbine developed by Aquascientific Ltd. and Exeter University. The MRL turbine is designed to work in shallow waters so it can utilise the potential of high speed flow close to the surface. The turbine operates with three straight blades of constant span-wise section, as shown in Figure 1. A key design featured of the MRL turbine is that it relies on the combination of both lift and momentum reversal (drag) for energy extraction. Drag forces are generally less sensitive to flow direction than lift forces. Additionally, cross flow turbines benefit from the fact that bending moments of the blades are reduced compared with those in conventional horizontal axis turbines. Preliminary test campaigns were undertaken in a high blockage ratio wind tunnel and re-circulating flume tank [15]. It was also found during those initial test campaigns that the implementation of non-linear blade locus improved the efficiency of the device as the contributions of lift and drag forces were optimised with different blade rotation stages. Similarly, it was demonstrated that the MRL turbine benefited from the use of sided plates (Figure 1) which increased the 'Pelton effect' from the top plate and similarly generated a 'ground effect' from the bottom plate.

The blades of the MRL rotate around a common central horizontal axis which is parallel to their own 

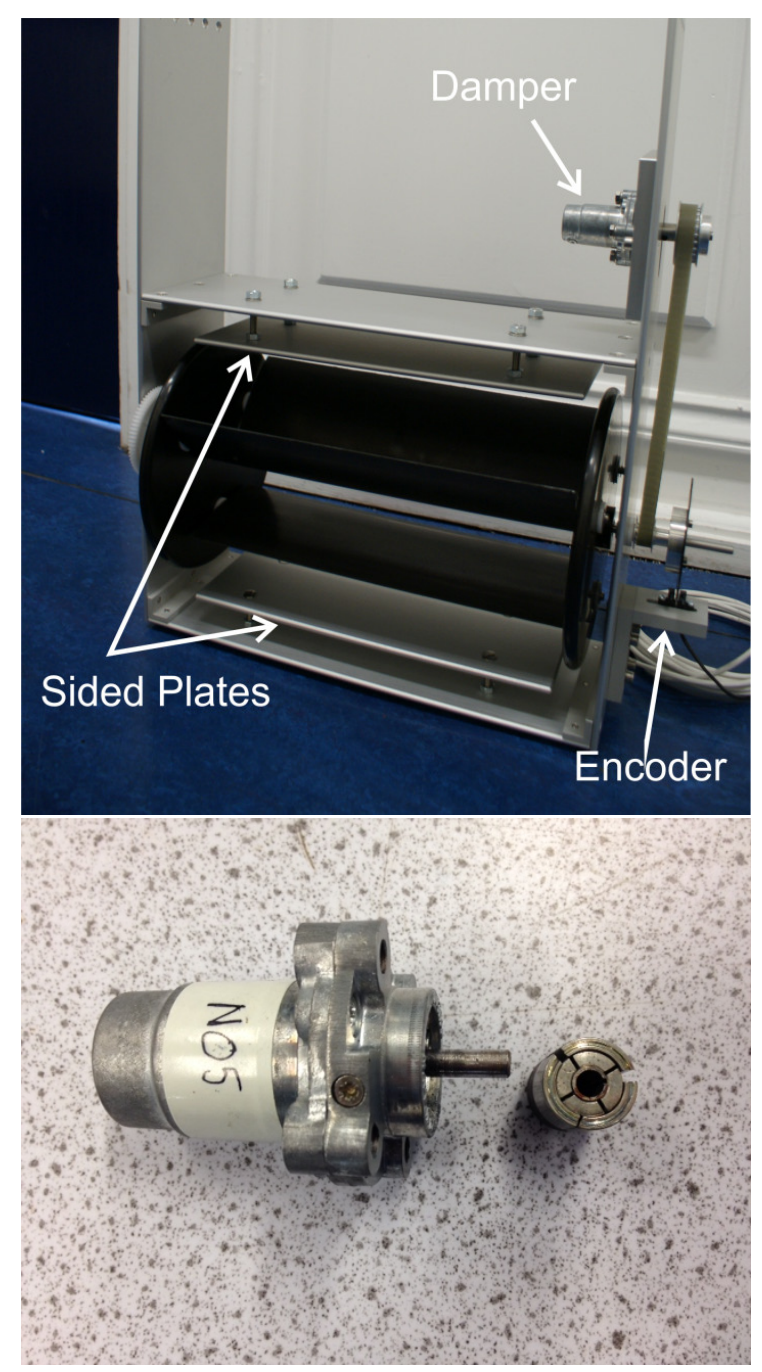

Figure 1: MRL Turbine (top) and the damper used in the experiments (bottom)

axis and perpendicular to the flow. The pitch of each blade changes as the turbine rotates. Every time the main shaft completes a full rotation, each blade revolves by $180^{\circ}$ independently from the main axis (i.e. each turbine rotates about their own axis). Four small scale MRL turbine models were used in these experiments with dimensions of: $0.095 \mathrm{~m}$ blade chord, $0.164 \mathrm{~m}$ diameter (D) (taking into account blade angles to the onset flow) and $0.3 \mathrm{~m}$ blade span (L), as depicted in Figure 2. Power take off is simulated using an oil filled dashpot, attached to the main shaft via a gear and pulley system.

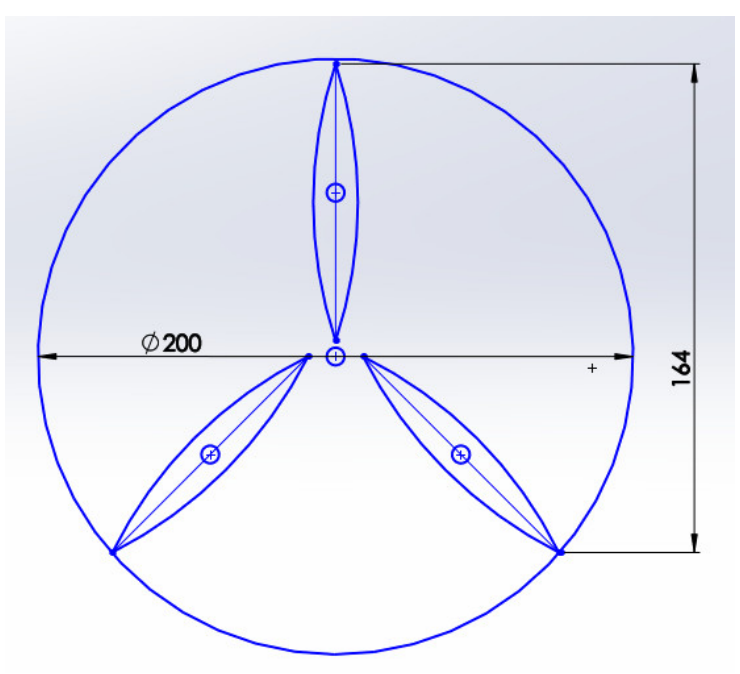

Figure 2: Side view of the MRL Turbine showing the blade angles to the onset flow.

\subsection{Damper Calibration and Power Output}

The turbine model's resistive torque could be varied by the configuration of the dashpot and gearing system. The damping of a dashpot is dictated by an internal surface moving through a viscous fluid. The torque $(\tau)$ required to turn the damper at a given rotational velocity $(\omega)$ was investigated with a torque test rig. The rig consisted mainly of a motor, a rotational velocity sensor, a torque transducer and a damper. Three different dampers with the following viscosities were tested: $1,2.5$ and $5 \mathrm{kSt}$ [16]. The DC motor was rated at $5 \mathrm{Nm}, 147 \mathrm{rpm}$ and $27 \mathrm{~W}[17]$ and was selected based on price and loading requirements. A contactless rotary torque transducer with a rated capacity of $2 \mathrm{Nm}$ was used for the calibration procedure [18]. The torque rig was equipped with a digital Hall effect vane sensor and a 24 tabbed disc to measure angular speed.

Once the relationship between $\tau$ and $\omega$ is quantified for each damper - gearing configuration, the power extracted by the turbine can be calculated via:

$$
P_{\text {captured }}=\omega_{d} \tau
$$

where $P$ is the power generated by the MRL turbine, $\omega_{d}$ corresponds to the angular velocity of the dashpot, inferred from the main shaft rotational velocity and the gearing ratio used (Figure 1).

The efficiency of a turbine is defined as the proportion of the available power in the fluid captured. In order to do this the power in the flow across the 

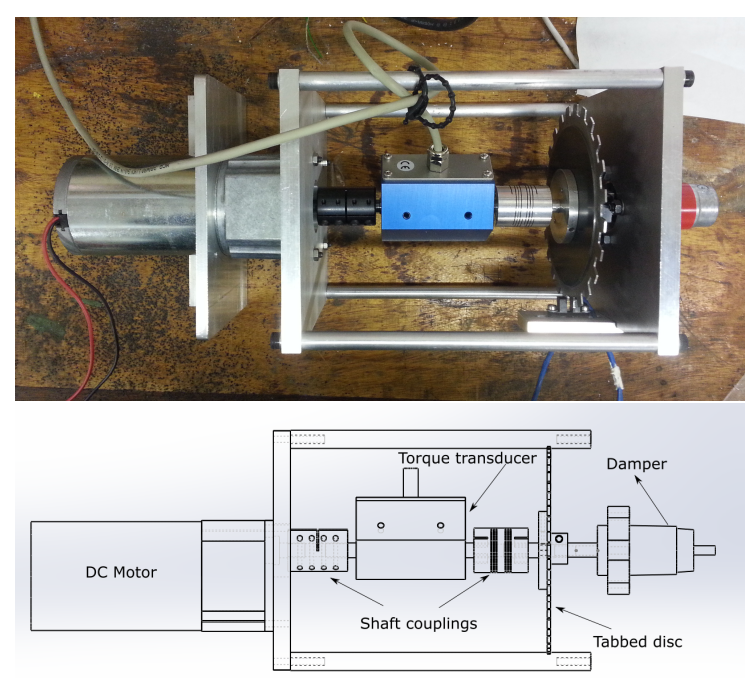

Figure 3: Turbine damper calibration rig (top) and schematic side view (bottom)

swept area $(A)$ is:

$$
P_{\text {flow }}=0.5 \rho A u^{3}
$$

where $\rho$ represents the density of water and $u$ the measured upstream flow velocity. For the MRL turbine, the swept area is $A=D \times L$. Thus the power coefficient $\left(c_{p}\right)$ of the turbine for a given configuration is given by:

$$
c_{p}=\frac{P_{\text {captured }}}{P_{\text {flow }}}
$$

In a HATT device the coefficient $c_{p}$ is normally related to the local rotational velocity of the rotor through the non-dimensional parameter called Tip Speed Ratio. Due to the geometry of the MRL turbine, the blade speed ratio (BSR) is used instead to establish a similar relationship, defined as follows:

$$
B S R=\frac{\omega R}{u}
$$

where $\omega$ is the angular velocity around the main shaft of the turbine in $\mathrm{rad} / \mathrm{s}$ and $R$ is the separation between the central shaft and the centre of rotation of the individual blades, equal to $0.055 \mathrm{~m}$ here.

\subsection{Flow Measurement}

The test campaign was conducted in the WaveCurrent Circulation tank at IFREMER, Boulogne sur Mer, France. The channel's dimensions are 4

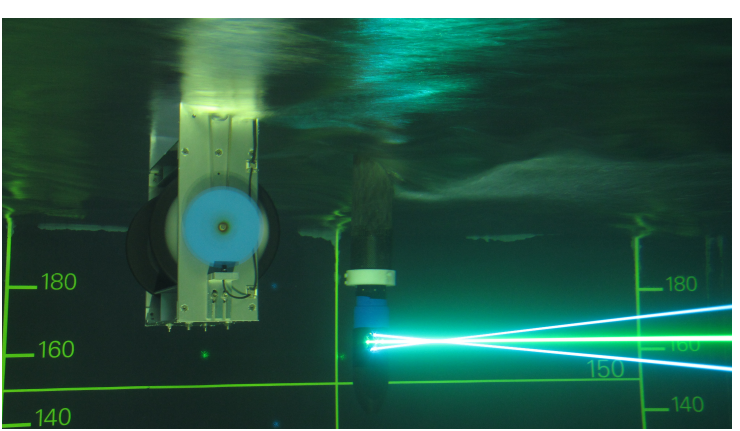

Figure 4: MRL turbine (centre-left) mounted in the WaveCurrent Circulation tank. On the right hand side the LDV is taking measurements of the wake developed by the turbine located on the left hand side.

$\mathrm{m}$ wide, $2 \mathrm{~m}$ deep and $18 \mathrm{~m}$ in length. As the MRL turbine was specially designed to be utilised in shallow to medium flow depth estuaries, the turbine was installed with the main shaft $1.1 \mathrm{D}$ below the water surface, as depicted in Figure 4. This was to reproduce accurate blockage effects at the free surface. The turbine was rigidly mounted onto the top of the tank using $\mathrm{H}$-beams available at the facility. For the single turbine experiments, the device was situated in the midsection of the tank. In the arrays the turbines were mounted symmetrically around the midsection.

The wake generated by a single turbine was characterised by measuring velocity at a range of points downstream of the turbine. A 2D Laser Doppler Velocimeter (LDV) was mounted on a computer controlled gantry was used to measure velocity across the wake, thus building up a velocity grid. The velocity grids are represented by two velocity components $u$ and $v$. The $u$ velocity vector is along the streamwise $(x)$ direction (positive in the flow direction) and the $v$ velocity vector is the transverse $(y)$ component (following the right hand rule). Figure 4 , shows the LDV probe during operation.

The turbine was operated in a current of nominally $1.0 \mathrm{~m} / \mathrm{s}$ with nominal turbulence intensity of around $3 \%$ controlled via a series of upstream screens, giving a Reynolds number of $8.2 \times 10^{4}$. The length scale at the rotor centre was $0.293 \mathrm{~m}$ and was calculated according to [19]. The wake structure for MRL turbine arrays were measured along a grid described in an ' $x, y, z$ ' coordinate system. The origin was set at the centre of the turbine rotor of the first row and at the lateral centreline of the tank. The velocity at each point was measured for 100 s. 
The two metrics used here to characterise the flow are the mean stream-wise velocity deficit $(\Delta u)$ and the turbulence intensity $(I)$. Both these metrics are defined over a period of statistical stationarity of 100 seconds. The velocity deficit at a given point in $x$ is defined by:

$$
\Delta u=100 \cdot\left(1-\frac{\overline{u_{x}}}{u_{0}}\right)
$$

where $\overline{u_{x}}$ is the mean velocity at a given point and $u_{0}$ is the velocity measured at the array location with the same flume settings, but without the turbines installed.

The turbulence intensity is defined as the root mean square velocity fluctuations (that is the absolute value of the instantaneous velocity fluctuation minus the mean) normalised by the mean velocity at that point expressed as a percentage:

$$
I=100 \cdot \sqrt{\frac{\frac{1}{2}\left(\left\langle u^{\prime 2}\right\rangle+\left\langle v^{\prime 2}\right\rangle\right)}{\bar{u}}}
$$

where $\bar{u}$ is the mean streamwise velocity, $u^{\prime}$ is the streamwise velocity fluctuation, $v^{\prime}$ is the transverse velocity fluctuation and angled brackets indicate the mean value over the stationarity period. Note that the vertical contribution to $I$ is not included because the LDV system was not capable of measuring the vertical velocity component. The LDV measured flow velocities within small confidence intervals of $+0.12 \mathrm{~m} / \mathrm{s}$ for a $95 \%$ of confidence level and an average standard deviation of $0.06 \mathrm{~m} / \mathrm{s}$.

\section{Array Layouts}

A single turbine and three array setting were studied during this test campaign. The arrays included in this study were: a staggered array of four turbines ('Staggered'), a tandem array of three Turbines ('Tandem') and an aligned array of three turbines in a fence ('Fence'). The spacing between turbines laterally and longitudinally in each of the arrays are given in Table 1. The number of downstream velocity locations measured was varied according to the array and is detailed in the appropriate section below.

\subsection{Single Turbine}

The wake characteristics of the single turbine were explored first in order to obtain high resolution measurements and guide future array spacings (Figure 5).
Table 1: Array layout edge to edge spacings used in the tests.

\begin{tabular}{llccc}
\hline Layout & Devices & $\begin{array}{c}\text { Row 1-2 } \\
\text { spacing }\end{array}$ & $\begin{array}{c}\text { Row 2-3 } \\
\text { spacing }\end{array}$ & $\begin{array}{c}\text { Cross-flow } \\
\text { spacing }\end{array}$ \\
\hline 'Staggered' & 4 & $10 D$ & $15 D$ & $5 D$ \\
'Tandem' & 3 & $10 D$ & $15 D$ & $\mathrm{~N} / \mathrm{A}$ \\
'Fence' & 3 & $\mathrm{~N} / \mathrm{A}$ & $\mathrm{N} / \mathrm{A}$ & $3 D$ \\
\hline
\end{tabular}

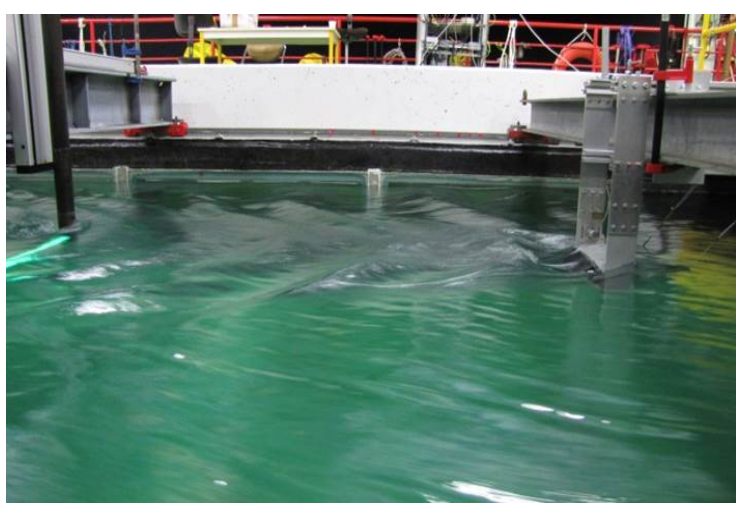

Figure 5: MRL turbine mounted in the Wave-Current Circulation tank.

Velocity measurements were taken across downstream planes at $x=:-2 D,-1.5 D,-1 D, 1 D, 1.5 D$, $2 D, 2.5 D, 3 D, 4 D, 6 D, 8 D, 10 D, 15 D$ and $20 D$. At each $x$ location 7 points were measured laterally (in $y$ ) at $0 D, 0.45 D, 0.9 D$ and $1.5 D$ either side of the turbine centreline totalling 7 locations). In addition, 4 vertical locations $(z)$ were measured at $z / D=0,0.6,1.2$ and 1.8 for each $y$ and $x$ location, giving a total of 420 velocity measurement points, as shown in (Figure 6). The blockage ratio for a single turbine (i.e. the swept area divided by the tank cross section) is $0.6 \%$.

\subsection{Staggered Array of Four Turbines}

The 'Staggered' array (Figure 7) consisted of four turbines installed in three rows. The lateral separation between the devices in the second row was $5 \mathrm{D}$. The stream-wise separations between rows 1 and between rows 2 and 3 was $10 \mathrm{D}$ and $15 \mathrm{D}$ respectively. 1064 velocity points were measured across each of the downstream planes at $x=:-1 \mathrm{D},-1.5 \mathrm{D}$, $1 \mathrm{D}, 1.5 \mathrm{D}, 2 \mathrm{D}, 2.5 \mathrm{D}, 3 \mathrm{D}, 3.5 \mathrm{D}, 4 \mathrm{D}, 6 \mathrm{D}, 8 \mathrm{D}, 10 \mathrm{D}$ for the first row and an additional 15D for the second and third row. Seven lateral measurements were taken at $1.25 \mathrm{D}$ separation from $-3.75 \mathrm{D}$ to $3.75 \mathrm{D}$. Vertical measurements were taken at $z / D=0,0.5$, $1 \& 1.5 D$. 


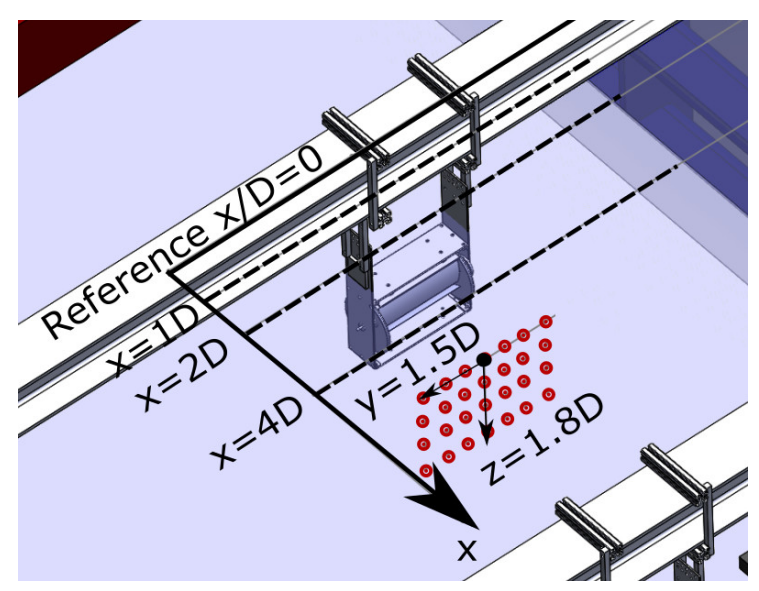

Figure 6: Grid showing the velocity points taken at 1D for the MRL single turbine experiment. The mesh shows the distances of $1 \mathrm{D}, 2 \mathrm{D}$ and $4 \mathrm{D}$ in the downstream direction $(x)$. The lateral and vertical direction is represented by $y$ and $z$ respectively.
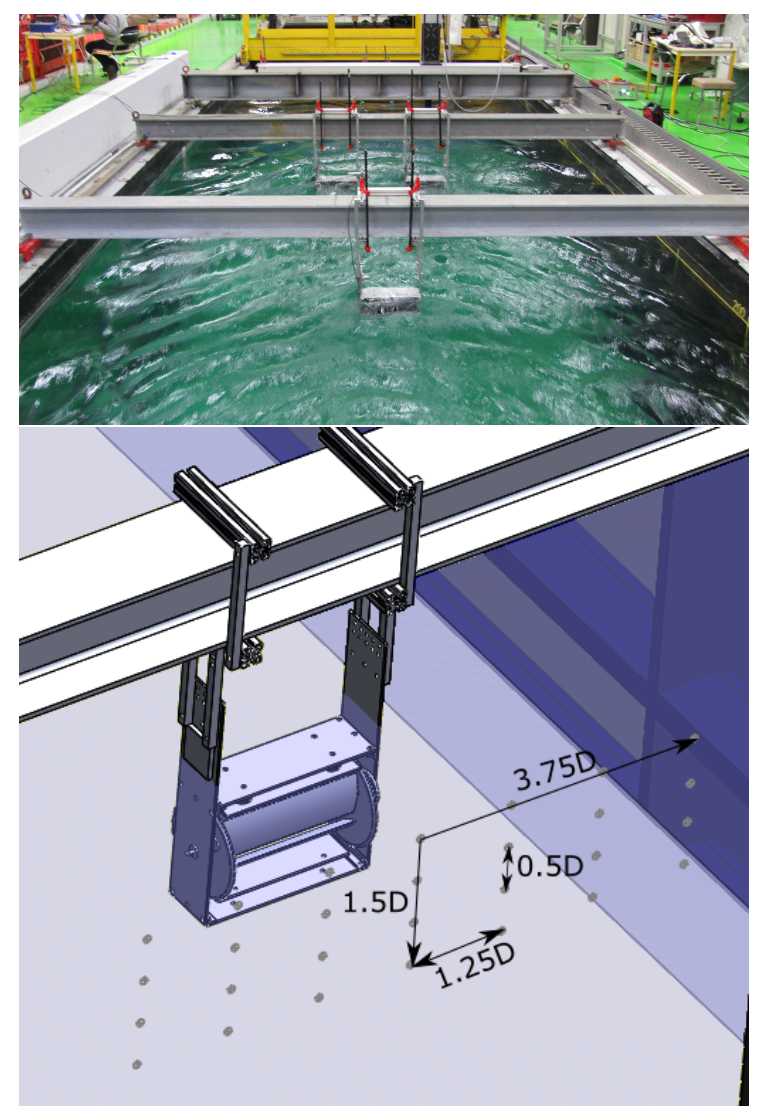

Figure 7: Staggered Array of Four Turbines mounted in the Wave-Current Circulation tank (top), the second figure shows the velocity grid (bottom). The fourth turbine is in alignment with the turbine at the front of the array configuration at $25 \mathrm{D}$ downstream.

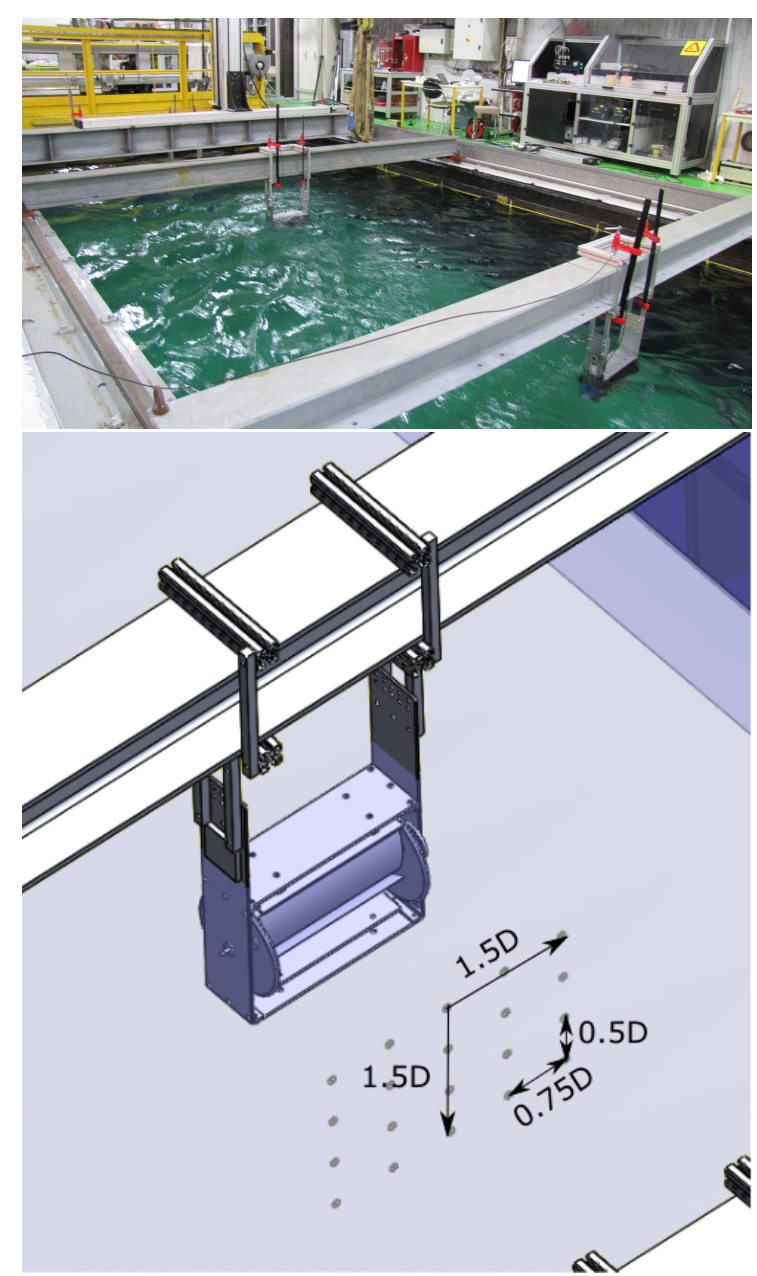

Figure 8: Tandem Array of Three Turbines mounted in the Wave-Current Circulation tank (top), the second figure shows the velocity grid (bottom). The third turbine is in alignment with the turbine at the front of the array configuration at $25 \mathrm{D}$ downstream.

\subsection{Tandem Array of Three Turbines}

The 'Tandem' array (Figure 8) consisted of three turbines aligned along the $y$ axis. The separation between row 1 and 2 was $10 D$ and between row 2 and 3 was $15 D$. Velocities were measured across each of the downstream wake planes as per the 'Staggered' configuration. In the transverse direction $(y)$, velocities were measured at $y=:-1.5 \mathrm{D}$, $-0.75 \mathrm{D}, 0,0.75 \mathrm{D}$ and $1.5 \mathrm{D}$. The vertical spacings were kept the same as for the other arrays. Thus the number of points measured across the 'Tandem' array were 760 . 


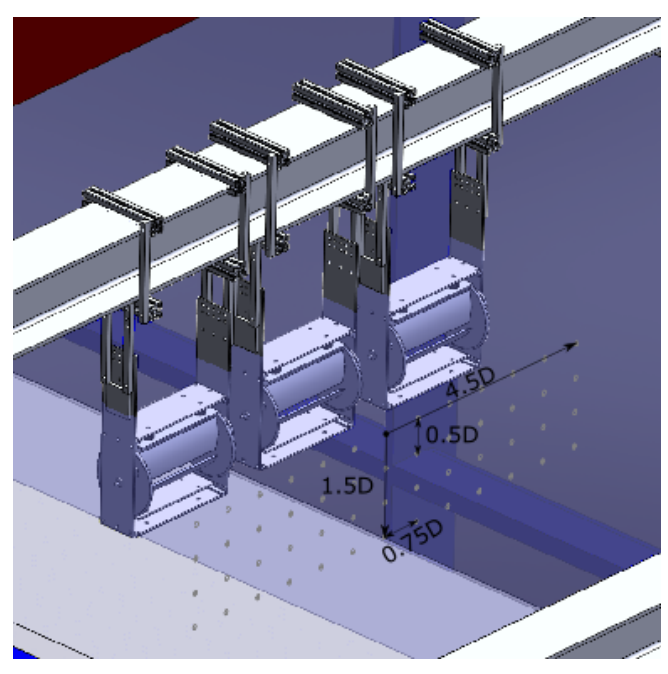

Figure 9: Aligned formation of turbines of the 'Fence' array showing also the velocity grid.

\subsection{Three-Turbine Fence}

The final array tested in this campaign consisted of three turbines aligned along the $x$ axis with a separation of $3 D$ (Figure 9). 14 downstream measurement planes along the $x$ direction were taken during the trials, as for the 'Tandem' array but adding a plane at 20D with 13 locations at each streamwise location in the $y$ axis at a spacing of $0.75 \mathrm{D}$ about the centreline. For this array velocity points were only measured at a single elevation of $z / D=0$, giving a total of 182 measurement locations.

\section{Results and Discussion}

For the arrays, velocity profiles and turbulence intensities are presented for each of the layouts. Evolution of the flow into and within the wake of the turbines are presented via maps of mean velocity magnitude $\left(\sqrt{\left(\bar{u}^{2}\right)+/\left(\bar{v}^{2}\right)}\right)$ profiles and velocity deficits downstream of the turbine. The fluctuations in the flow velocity are shown as turbulence intensity maps.

\subsection{Turbine Damper and Gearing Calibration}

Figure 10 shows the performance curves of the single MRL turbine in seven different damper and gearing configurations. As can be seen the $2.5 \mathrm{kSt}$ damper with on a 24:12 gear ratio produced the highest $c_{p}$ values. Thus the configuration $2.5 \mathrm{kSt}$ damper with on a 24:12 gear ratio was used for all subsequent experimental work. Figure 10 was obtained with a large separation between the ground

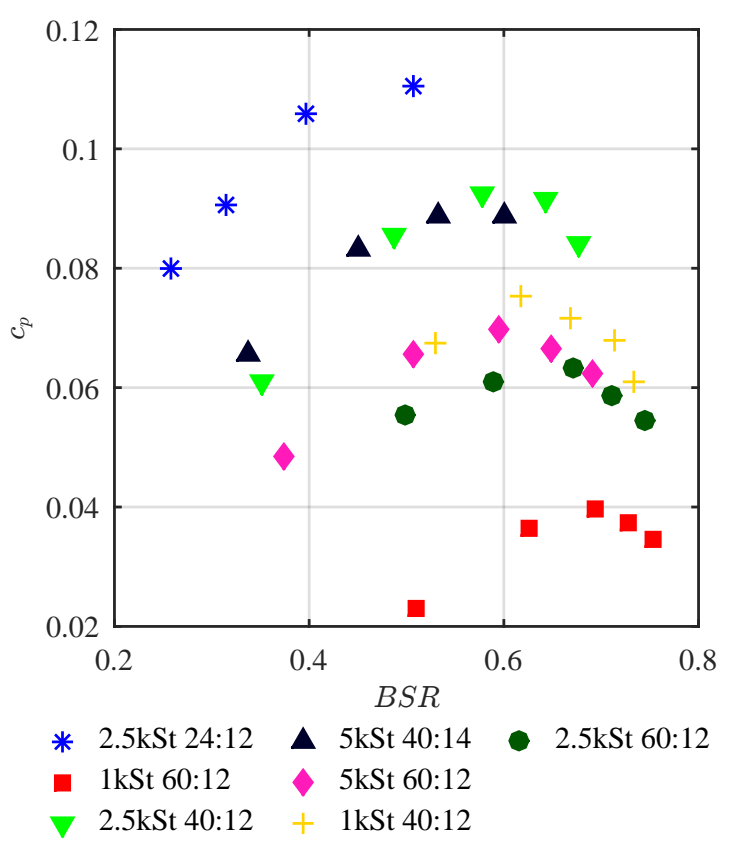

Figure 10: MRL Performance for a range of Damper and gear ratio configurations. For each case, the legend shows the damper viscosity (in kst) and the gear ratio.

effect plate and the blade. In subsequent tests presented in this paper a decreased of this distance lead to a $c_{p}$ increase of about $70 \%$, which was the configuration used for the remainder of this work.

It can be observed that this $c_{p}$ is of very low magnitudes compared to a generic HATT, [11] and [13] reported an average peak $c_{p}$ of 0.38 . Subsequent work done by Sutherland et al. [20] and carried out after these set of tests showed that combining the frictional torque of the damper with that of the turbine increases the $c_{p}$ up to about 0.25 . This values is still significantly lower than those shown by HATTs.

\subsection{Wake Characteristics of a Single Turbine}

Figure 11 shows the average axial velocity and turbulence intensity into and within the wake of a single MRL turbine at $z / D=0$. The velocity deficit peaks in the near wake region with an associated increase in $I$ as would be expected. The maximum value of velocity deficit along the $x=0$ axis is at $3 D$ where the deficit is $50-60 \%$. The deficit decreases steadily and monotonically to approximately $24 \%$ at $20 \mathrm{D}$, the furthest downstream measurement. This recovery is slow compared to HATTs in similar tests, which showed lower values of velocity deficit at $10 D$. However, in the 

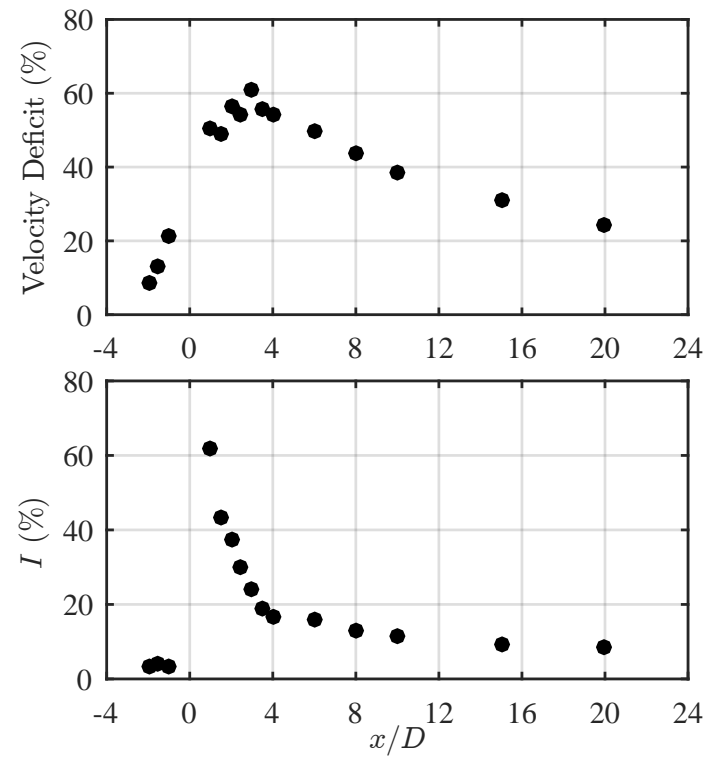

Figure 11: Velocity deficit and turbulence intensity evolution through a single MRL turbine.

near wake, the velocity deficit is slightly lower than in the HATT experiment with similar levels of $I$ [21]. This difference in velocity deficit could be attributed to the low Reynolds numbers obtained here compared to the ones showed in [21] which were in the order of $10^{5}$, as discussed in [22]. Also, it can be observed upstream $(x=-2 D$ to $-1 D)$ of the turbine, that the presence of the MRL turbine significantly decreases the inflow in the range of 10 to $20 \%$ compared with the nominal tank velocity $\left(u_{0}\right)$ with no turbine installed. The turbulence intensity is less affected in the upstream area, preserving values of $3 \%$ which is the nominal undisturbed value developed by the flume. $I$ is highest at $1 D$ in the $x=0$ axis, reaching a value of about $60 \%$ and then decreasing rapidly until approximately $8.5 \%$ in the region of $15-20 D$.

Figure 12 shows the resultant velocity, flow direction and turbulence intensity across the $z / D=0$ plane for the single MRL turbine. The lowest velocity and associated highest $I$ values for this region are at the edges of the device $(y / D \approx 0.91)$ $1 D$ downstream. This is also where the flow direction deviates from the stream-wise by the greatest amount. $\bar{u}$ has a minimum value of $<0.28 \mathrm{~m} / \mathrm{s}$ and $I$ a maximum of $>55 \%$. There is a degree of asymmetry in the near wake with the velocity slowing more in the $y / D>0$ values. This is likely to be due to the asymmetry of the device where the power take off system is located in this side of the turbine but also due to a directionality of the upstream flow towards that direction, as shown in Figure 11 at $x / D<0$. $I$ then decreases significantly between $1 D$ and $4 D$ downstream at the edges of the turbine. For the majority of the region $8 D-20 D$, $I$ is in the range of $8-10 \%$ but never recovering the upstream $I$ of $3 \%$.

The wake expands laterally in almost all the measured region, reaching values of $y / D=1.5$ which seems to expand more than with HATTs as shown in [21], where the non-dimensional values reaches only to $1 \mathrm{D}$ and $-1 \mathrm{D}$. As before, this could be related to low Reynolds Numbers. Blockage factor is not being considered a substantial impact on the wake as in the experiments carried out for HATTs [21] and here, the blockage factor is minimal (less than $5 \%$ ). This is to some extent expected due to the geometry of the device and the rotational direction of the device. Again, it can be observed that the flow skews to the positive side region of the tank, suggesting that it is due to the effects from the measurement side of the turbine. The same is seen on the $I$ maps where the most turbulent region is located on the same side of the tank and mostly from 1-8D, which shows the effects of the pitching and measuring mechanism (Figure 1). An average $c_{p}$ of 0.10 was calculated for the MRL turbine with a power output of $2.1 \mathrm{~W}$.

Figure 13 shows the vertical variation of the flow under the turbine. There is a sharp gradient at the $1-2 D$ downstream region, as the flow is slowed directly behind the turbine but accelerates underneath. The wake then starts to expand with the gradient between $z / D=0$ and $z / D=1.5$ decreasing up to $20 D$. An additional view of the vertical profile can be seen in Figure 14 which is related to $x / D=3 D$.

\subsection{Wake Characteristics of the 'Staggered' Array}

In the staggered array case, the measured deficit of mean velocity along $y / D=z / D=0$ line of the first row turbine is found to be around $43 \%$ in the near wake of the rotor (1-2D downstream). This increases to about $50 \%$ at $3 D$ then reduces again to $40 \%$ by $10 D$, as shown in Figure 15 . The turbulence intensity decreases from 34 to $20 \%$ in the near wake (1-3D) and reduces to less than $10 \%$ when reaching row 2 . The flow is lower at $10 D$ than the values measured in the single MRL turbine case. This may in part be due to a turbine modification which decreased the separation between the lower 

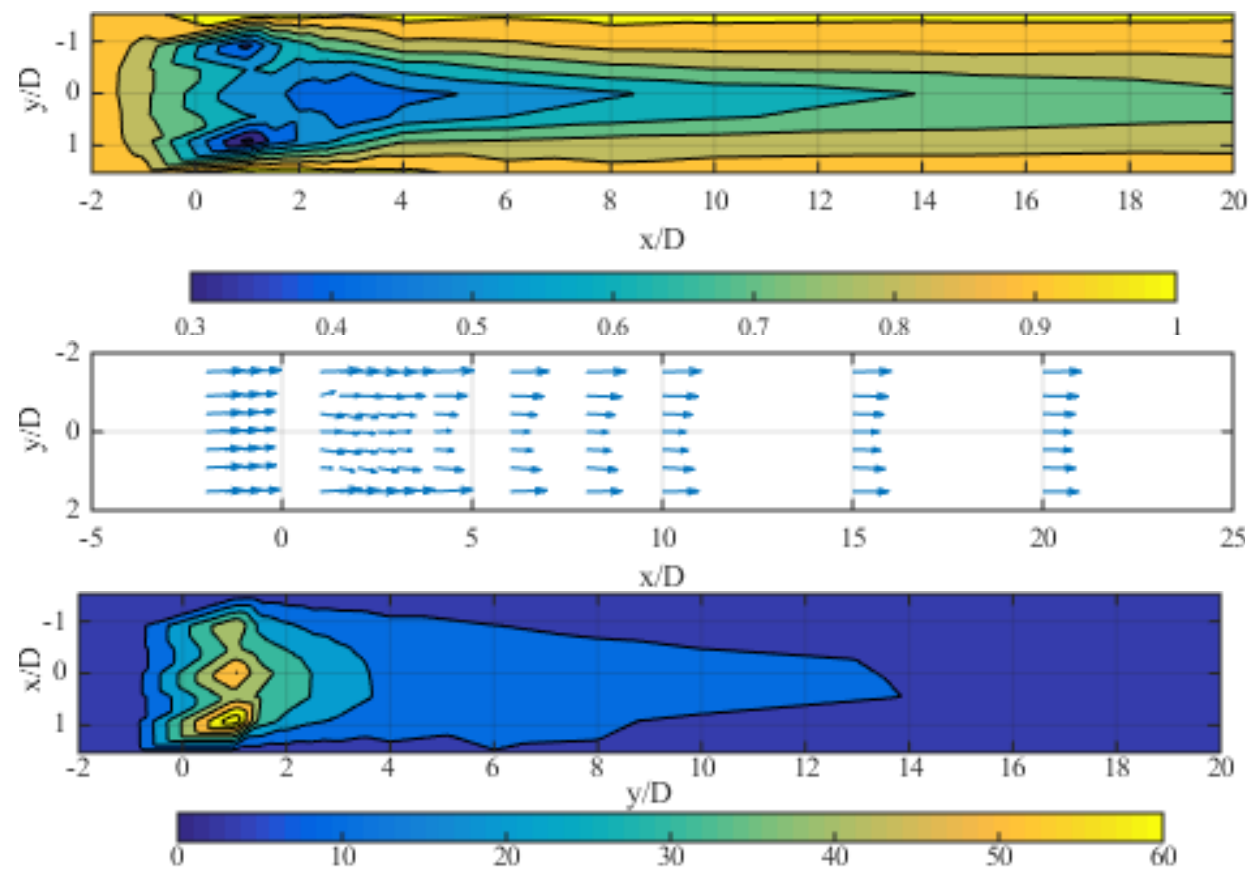

Figure 12: Axial velocity contour (top), velocity vectors (middle) and turbulence intensity (bottom) maps of a single MRL turbine. Axial velocity is presented as $\mathrm{m} / \mathrm{s}$ and turbulence intensity is given as (\%).
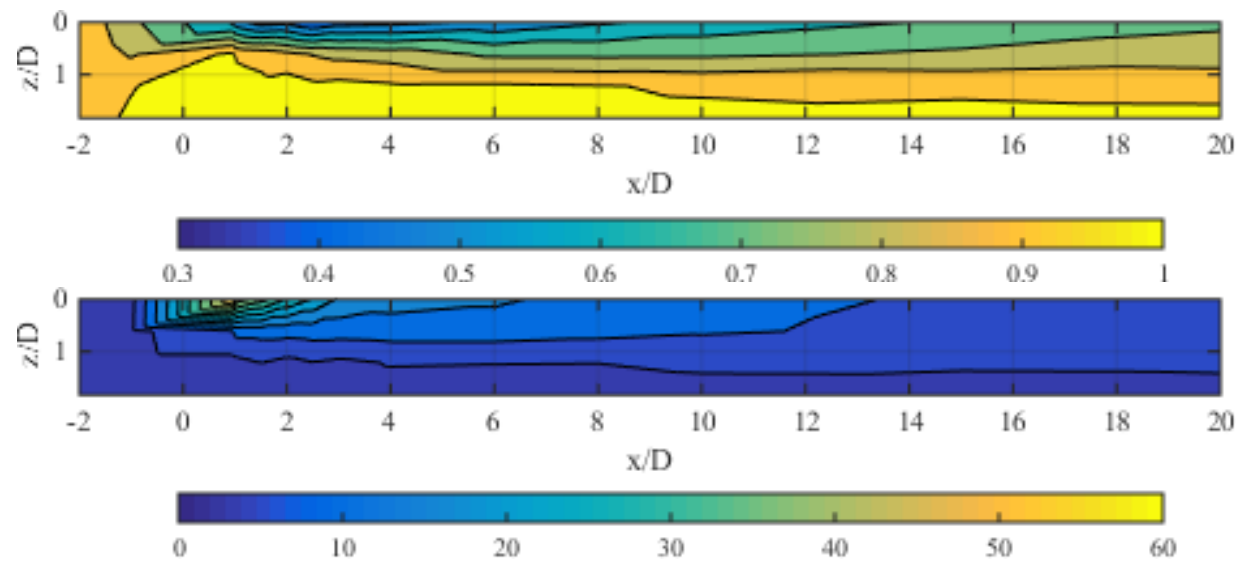

Figure 13: Vertical variation of the axial velocity and turbulence intensity along the $y / D=0$ centreline of the single turbine. Velocity deficit is presented as $\mathrm{m} / \mathrm{s}$ and turbulence intensity is given as (\%) 


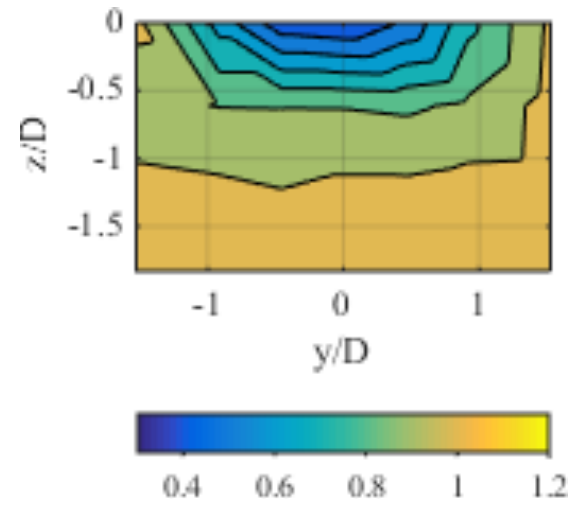

Figure 14: Measured axial velocity contour $(\mathrm{m} / \mathrm{s})$ of the flow under the turbine in the $y-z$ plane at $x / D=3 D$.
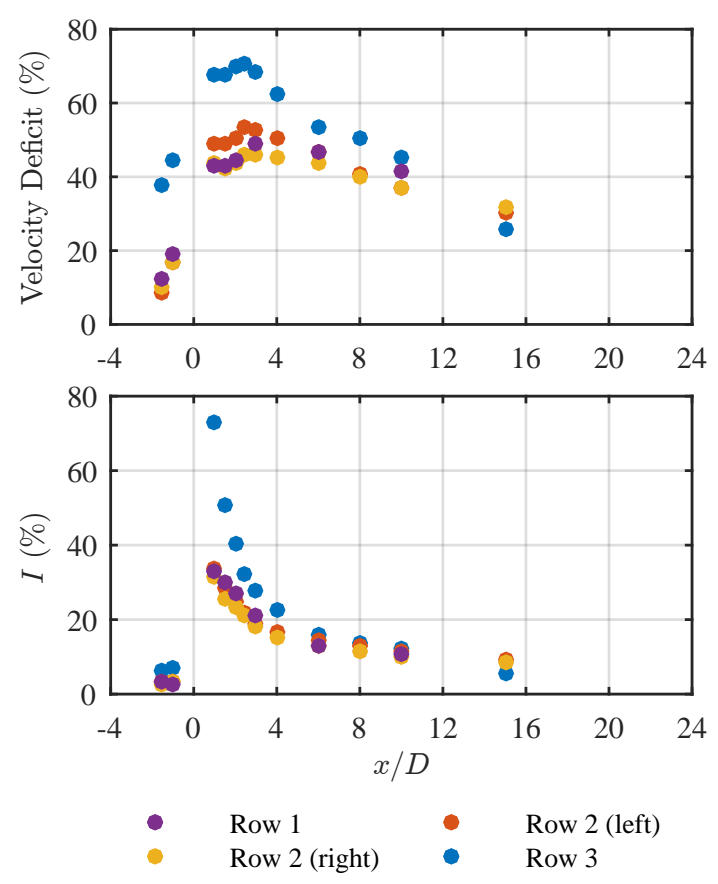

Figure 15: Velocity deficit and turbulence intensity that develops each of the $y / D=0$ centreline of the turbines on the 'Staggered' array. side plate and the blade. This had the effect of increasing the $c_{p}$ by $\sim 70 \%$ and the associated effect on the velocity was to increase the deficit and $I$ by $\sim 25 \%$ and $\sim 35 \%$ respectively in the near wake.

A similar set of behaviours is observed in Figure 16(middle) compared to the flow features of the turbine in row 1 , as the upstream flow is in a similar range of $0.8-0.9 \mathrm{~m} / \mathrm{s}$. In the wake of the second row the velocity deficit and turbulence intensity caused by the turbines is similar to that found in row 1 . The turbine on the $y / D>0$ side causes a significantly higher velocity deficit $(\sim 50 \%)$ in the near wake. This is related to the upstream conditions which are shown in the directional plot in Figure 12 to be skewed in the $y / D<0$ direction. However, despite the lower in-flow, the $c_{p}$ remains similar to both turbines, with a value of 0.15 . The performance of the most down-stream turbine is the lowest in the array with values of $c_{p}$ almost 2 times lower than those of the most upstream device. The velocity deficit across the 3 nd row turbine is $\sim 75 \%$ higher than across the others. This relates to the low upstream velocities that are developed after both rows 1 and 2. Overall, the velocity deficit recovers progressively after each row reaching values of $\sim 10 \%$ at $10-15 \mathrm{D}$. The turbulence intensity recovers more rapidly, reaching a value of $\sim 10 \%$ within $10-15 D$.

Figure 16(top) and Figure 16(middle) show the maps of the first row and second rows, respectively. It can be observed in Figure 16(middle) that the wake expands drastically compared to 16 (top) due to the two turbines. The wake of the two individual turbines in row 2 have merged into a single wake before reaching the third row. During the expansion there is a relatively unaffected area at $y / D=-3$ on one side of the array. The wake remains between $0.8-0.9 \mathrm{~m} / \mathrm{s}$ in the outermost part of the wake in the second and third row. By contrast, Turbine 2 (turbine on the $y / D>0$, row 2) generates a lower turbulence intensity in the near wake $(1-5 D)$. At its widest, the wake is evident across the entire measured area from the 2nd row of turbines till the extreme of the downstream measured area behind the 3rd turbine row.

There is again a degree of asymmetry in the wake with the effect on the downstream turbines being particularly noticeable. For example, comparing the inflow velocity upstream of the turbines in Figure 16(middle) and in the different $I$ values downstream of the same row in Figure 17(middle). The total mean power generated by this array was $9.7 \mathrm{~W}$ 

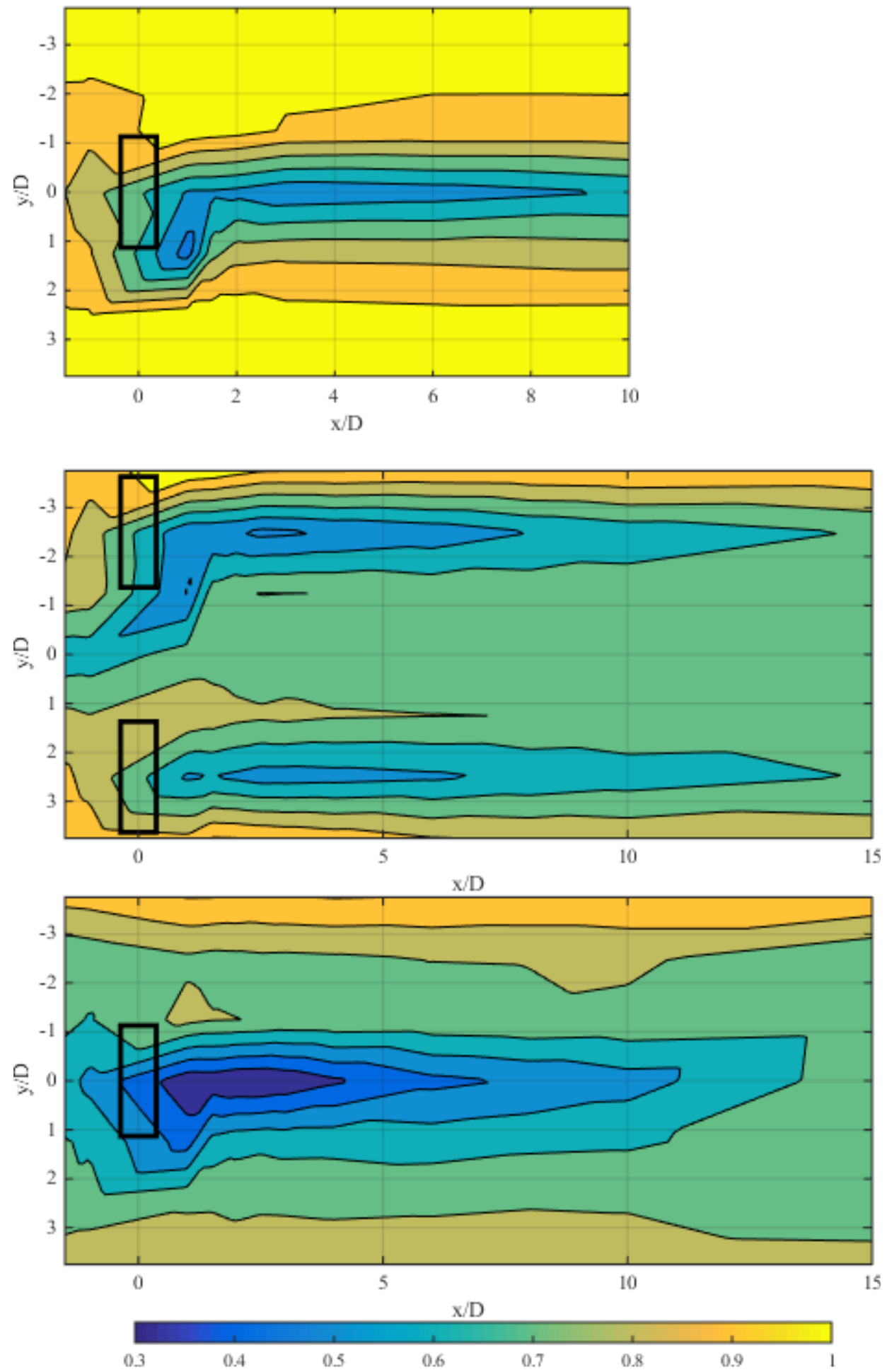

Figure 16: Axial velocity contours in m/s of the 'Staggered' array: Row 1 (top), Row 2 (middle) and Row 3 (bottom). 

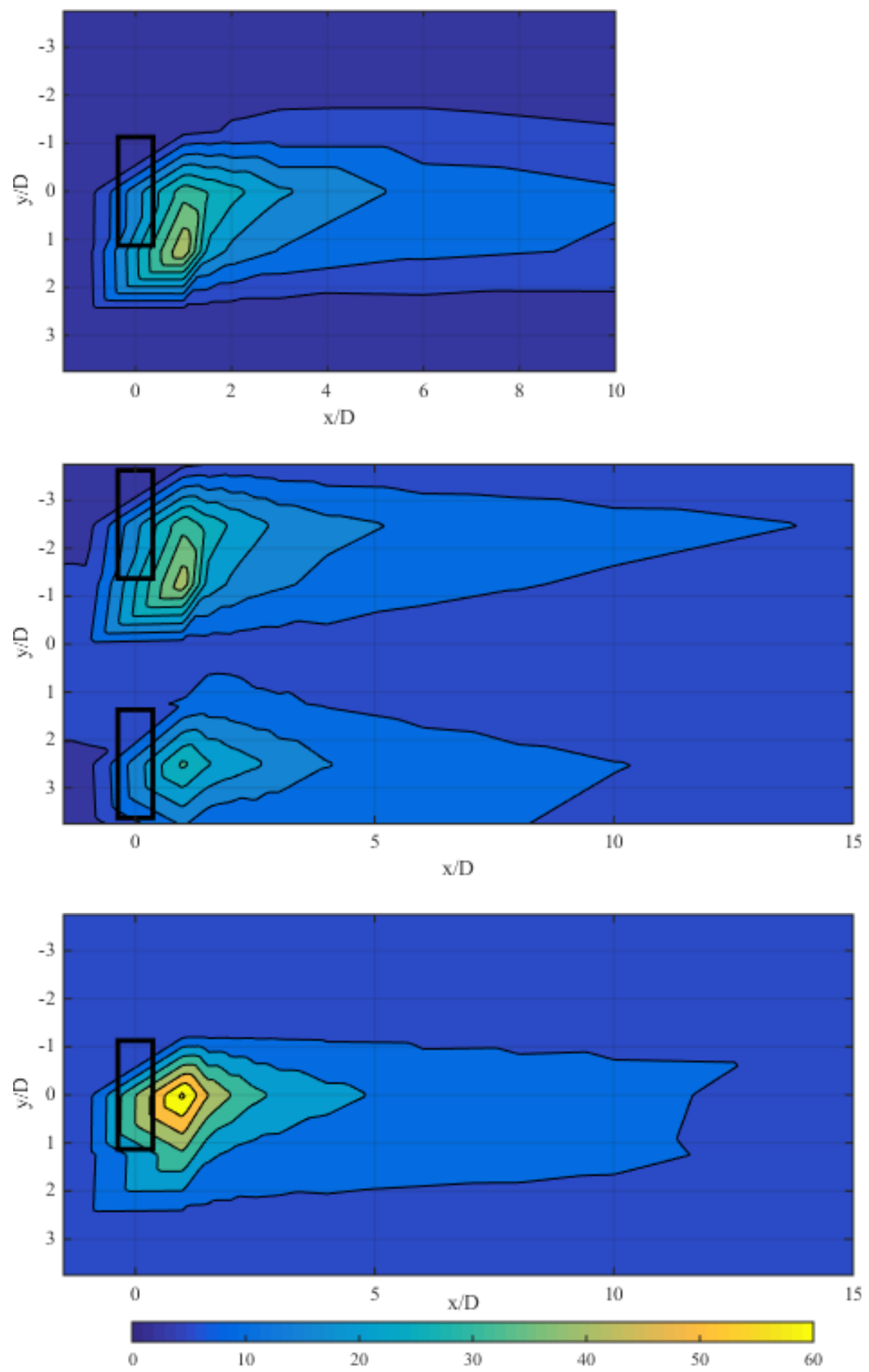

Figure 17: I map of the 'Staggered' array for Row 1 (top), Row 2 (middle) and Row 3 (bottom). Contour scale is in $\%$ 

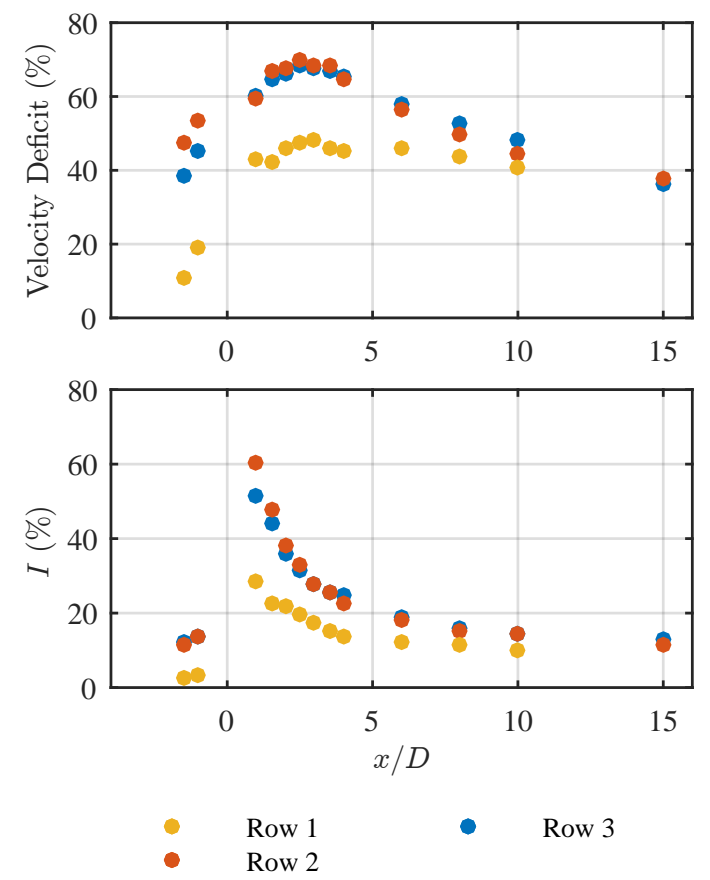

Figure 18: Velocity deficit and turbulence intensity that develops each of the centreline of the turbines on the 'Tandem' array.

or approximately $2.4 \mathrm{~W}$ per turbine on average.

\subsection{Wake Characteristics of 'Tandem' Array}

In the case of the Tandem array, the turbulence intensity in Figure 18 shows the highest $I$ values to be in the near wake, with values peaking at about $70 \%$ then recovering (decreasing) towards the upstream value. The $I$ values measured in the wake are similar to those reported in similar studies with HATT devices [11]. The turbulence intensity for the third row turbine has a maximum value $20 \%$ lower than that in the third row of the 'Staggered' array.

It is evident from Figure 19 that the most upstream turbine generates the lowest velocity deficit with the middle turbine generally the highest. This suggests that by row three, the mixing of the wake from the two combined upstream devices is leading to faster recovery. In general the two downstream devices show similar inflow velocities of $0.6 \mathrm{~m} / \mathrm{s}$ and $0.7 \mathrm{~m} / \mathrm{s}$ at $-1.5 \mathrm{D}$, respectively and similar wake recovery to a value of $40 \%$ at $15 \mathrm{D}$. The peak velocity deficit behind the turbines in Row 2 and 3 is 1.4 times higher than that developed by the first row. The velocity deficit values of the most upstream turbine are approximately equal to the single and first row of the 'Staggered' case.

Figure 20 show the lateral variation of $I$ in the array wake. In each case $I$, does not recover to its upstream value. It should be noted that this is in part due to the increased velocity deficit in the wake, by which $I$ is normalised by definition. This result is comparable with the previous array case. The $c_{p}$ from the upstream turbine $\left(c_{p}=0.16\right)$ is also similar to that of the equivalent device in the 'Staggered' array which was 0.18. The power coefficients $\left(c_{p}\right)$ for the downstream turbines remain at similar values of 0.13 and 0.16 despite the increased separation from Row 1 to Row 2 (10D) and Row 2 to Row 3 in this case. This shows that the turbine in the third row performs as well as that in the first, which suggests that a higher separation is beneficial between turbines. Here a slight increase of inflow velocity (from $0.6 \mathrm{~m} / \mathrm{s}$ to $0.7 \mathrm{~m} / \mathrm{s}$ ) results in a substantial increase in power $(>20 \%)$. An increase of $\mathrm{Cp}$ of about $60 \%$ should be expected due to the cubic relationship of power; however, the dashpots used in each turbine had slightly different characteristics and thus reflected in the power output between turbines. The results presented here are difficult to relate to others as array studies with power data are absent in the literature.

Again there is a degree of asymmetry in the wake with the $y / D>0$ side displaying greater velocity deficit and associated $I$. This effect appears to be compounded row by row. In the first row a small area at the extremes of the lateral measured range in unaffected by the turbine. By the second and third row the wake covers the entirety of the measured area. The total mean power output measured for this array configuration was $5.3 \mathrm{~W}$, representing on average $1.8 \mathrm{~W}$ per turbine.

\subsection{Wake Characteristics of 'Fence' Array}

The results obtained for the three-turbine fence show similar values of velocity deficit and $I$. The Turbine on $y / D>0$ presented larger discrepancies developing about $15 \%$ higher velocity deficits in the near wake, as seen in Figure 21. Figure 22 highlights the velocity acceleration between devices. The effect is significantly more than for the second row of the 'Staggered' array where the turbine separation was greater. The turbulence intensity is shown (Figure 23) to develop higher values in the turbines on the extremes than that in the middle. The $c_{p}$ developed by the outer turbines is the same, $c_{p}=0.19$, and the centre turbine is slightly lower at 

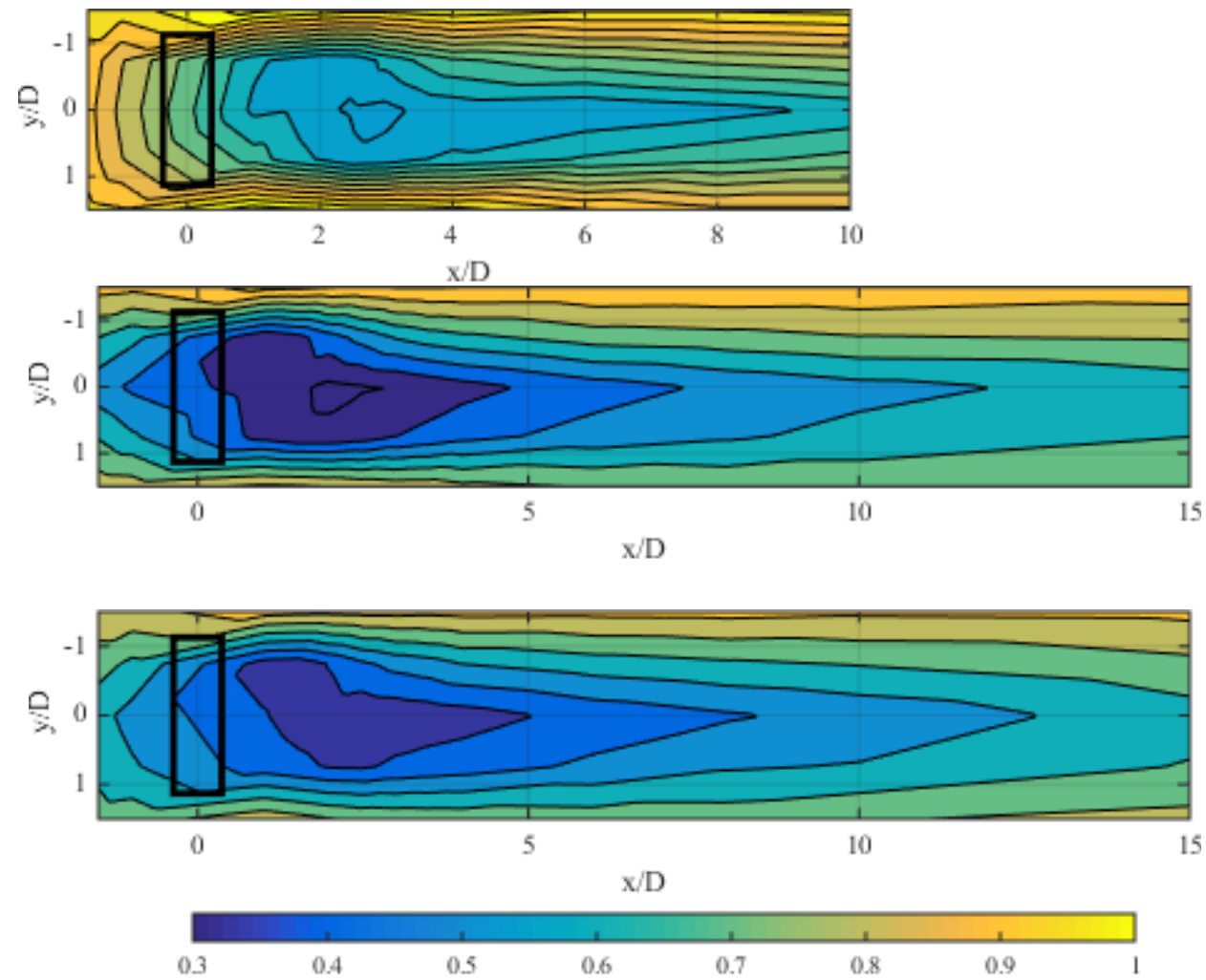

Figure 19: Axial velocity contour in m/s of the 'Tandem' array: Row 1 (top), Row 2 (middle) and Row 3 (bottom). 

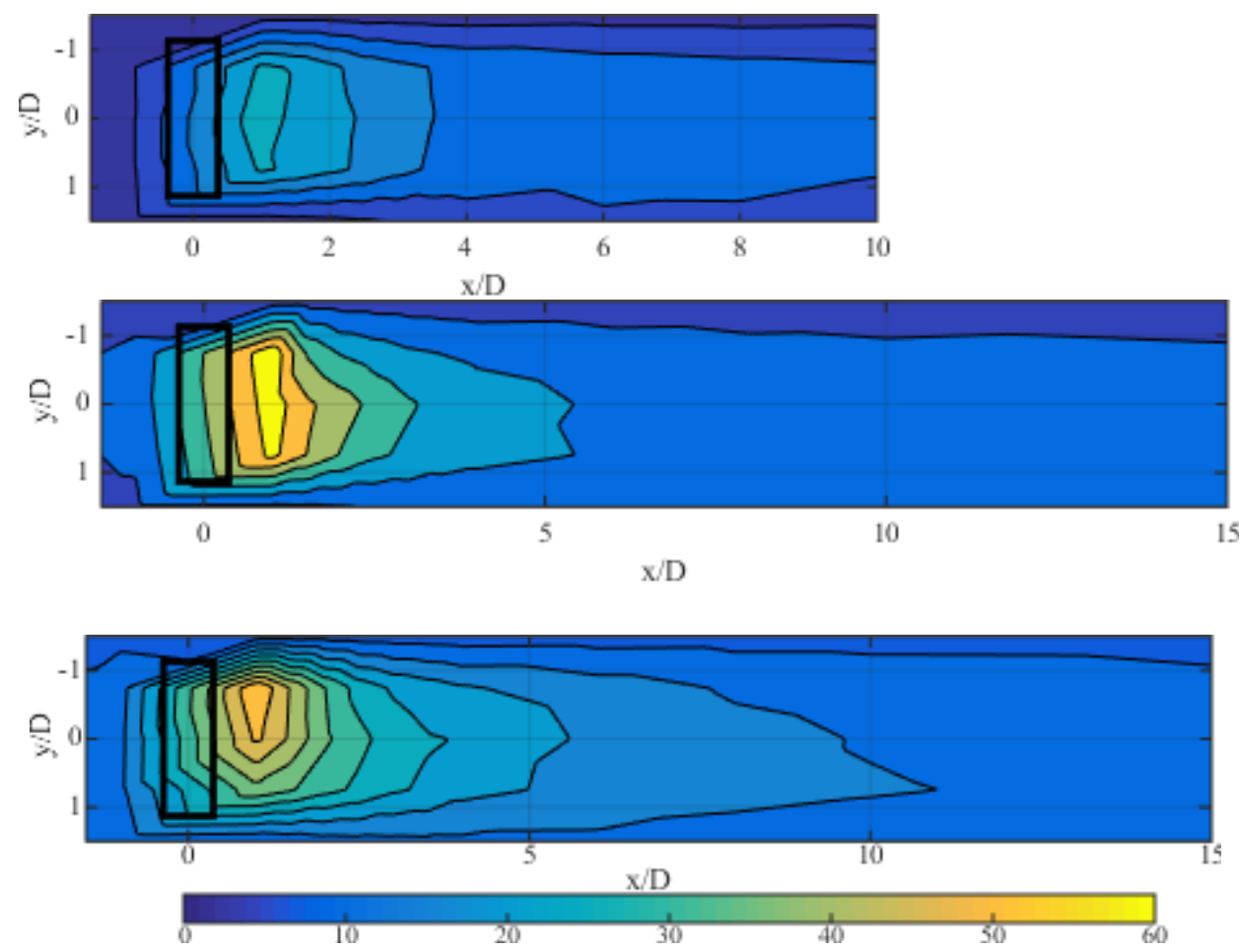

Figure 20: I map of the 'Tandem' array: Row 1 (top), Row 2 (middle) and Row 3 (bottom). Contour maps presented in $\%$.

$c_{p}=0.18$. However, the rotational speed reached by centre turbine is marginally higher $(106 \mathrm{rpm})$ than that on the extremes (103rpm and 98rpm). Therefore, the effect of the flow acceleration caused by the outer turbines is observed. The reduced $c_{p}$ can be attributed to small differences between turbines and dampers in terms of resistive torque used in these experiments, thus moving away from optimum damping conditions. These $c_{p}$ values are in line with those of the other array types.

For the three turbine fence, the asymmetry of flow effects is again evident. In Figure 22 it can be seen that the flow acceleration is greater and propagates more further downstream on $y / D>0$ than is the flow $y / D<0$. For the outer $y / D>0$ turbine the greatest velocity deficit is nearer to the turbine (at $2 D$ ) than for the other two at $3 D$. At the extreme edge on the $y / D<0$ the flow bypassing the array was observed to be greater than the opposite side. The wake does not recover to upstream velocities within the measured downstream area for this case as per the other array layouts. The total mean power output measured for this array layout was $10.1 \mathrm{~W}$ (3.3 W per turbine), and was the highest obtained across the array configurations investigated.

\section{Conclusions and Future Work}

An experimental campaign has been conducted to characterise the downstream wake of MRL turbine arrays. It was found that the velocity deficit of a MRL turbine is lower in the near wake but higher in the far wake compared to HATTs for comparable upstream $\bar{u}$ and $I$ values. Moreover, having a higher $c_{p}$ contributes also to a decrease in the velocity deficit.

In general it was found that the wake of the MRL turbines is not symmetrical but skewed to the $(y / D<0)$ side. This effect is independent of where the power take off mechanism is and thus it is associated with the fluid characteristics of the flume. In these experiments it was observed that the power take off mechanism only affects the near wake.

It was noticeable that the lateral expansion of the wake is higher in the MRL than for HATTs, reaching values of $1.5 \mathrm{D}$ whereas the HATT devices have a lateral expansion of up to 1D. Lateral expansion 

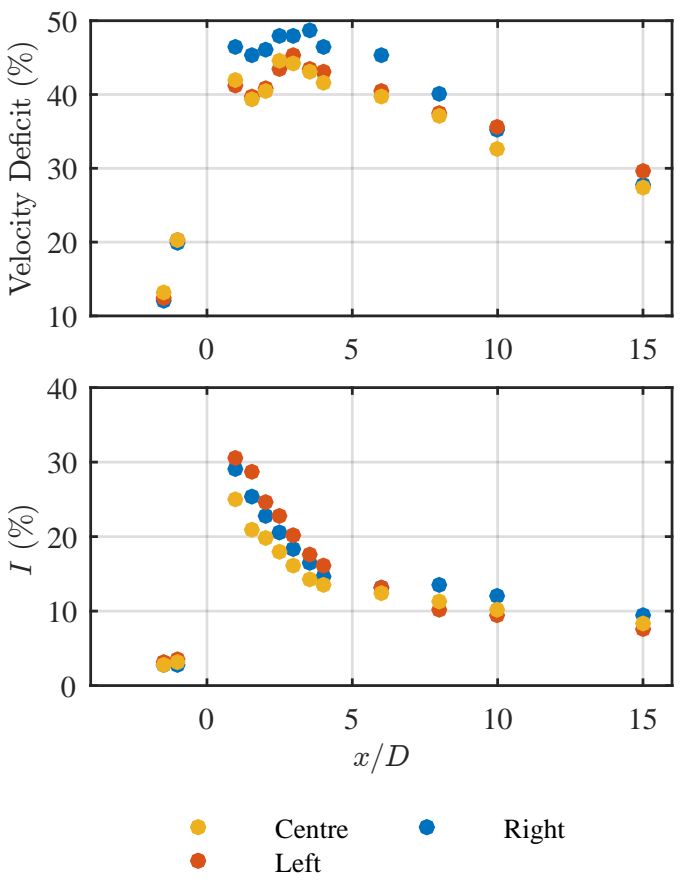

Figure 21: Velocity deficit and turbulence intensity that develops each of the centreline of the turbines on the 'Fence' array.

characteristics are mainly related to the geometry of the MRL turbine. The wake dissipates along the vertical direction up to $z / D=1.5$, being this the maximum expansion on the vertical at $x / D=3$ on the downstream direction. This lateral separation between devices across the investigated range $(3 D$ to $5 D$ ) does not seem to have a large effect on the far wake of devices even when there is a turbine upstream; i.e. the velocity deficit of the devices and the turbulence intensities recovers at the same proportion for both the 'Staggered' and 'Fence' arrays. Moreover, it was obtained that the fence array provided the highest power output out of the three arrays explored in this investigation. Thrust measurements were outside the scope of this study but will be considered in future research stages.

\section{Acknowledgements}

The authors would like to thank staff at IFREMER and at The University of Strathclyde for their assistance during testing. The authors would also like to acknowledge the EPSRC for funding this work under grant number: EP/J010138/1.

\section{References}

[1] M. Harrison, W. Batten, L. Myers, A. Bahaj, Comparison between CFD simulations and experiments for predicting the far wake of horizontal axis tidal turbines, IET Renewable Power Generation 4 (6) (2010) 613. doi:10.1049/iet-rpg.2009.0193.

URL http://digital-library.theiet.org/content/ journals/10.1049/iet-rpg. 2009.0193

[2] S. Draper, G. T. Houlsby, M. Oldfield, A. L. Borthwick, Modelling tidal energy extraction in a depth-averaged coastal domain, IET Renewable Power Generation 4 (6) (2010) 545. doi:10.1049/iet-rpg.2009.0196.

[3] T. Nishino, R. H. J. Willden, The efficiency of an array of tidal turbines partially blocking a wide channel, Journal of Fluid Mechanics 708 (2012) 596-606. doi: 10.1017/jfm.2012.349.

[4] T. A. A. Adcock, The available power from tidal stream turbines in the Pentland Firth, Proceedings of the Royal Society A: Mathematical, Physical and Engineering Science 469 (2157) (2013) 1471-2946. doi:10.1098/rspa.2013.0072.

URL http://rspa.royalsocietypublishing.org/ content $/ 469 / 2157 / 20130072$.short

[5] A. Olczak, T. Stallard, T. Feng, P. Stansby, Comparison of a RANS blade element model for tidal turbine arrays with laboratory scale measurements of wake velocity and rotor thrust, Journal of Fluids and Structures 64 (2016) 87-106. doi:10.1016/j.jfluidstructs. 2016. 04.001.

[6] M. G. Gebreslassie, G. R. Tabor, M. R. Belmont, N. P. Road, CFD simulations for investigating the wake states of a new class of tidal turbine.

[7] M. G. Gebreslassie, G. R. Tabor, M. R. Belmont, Numerical simulation of a new type of cross flow tidal turbine using OpenFOAM - Part I: Calibration of energy extraction, Renewable Energy 50 (2013) 994-1004. doi: 10.1016/j.renene.2012.08.065.

[8] M. G. Gebreslassie, G. R. Tabor, M. R. Belmont, Numerical simulation of a new type of cross flow tidal turbine using OpenFOAM - Part II: Investigation of turbine-to-turbine interaction, Renewable Energy 50 (2013) 1005-1013. doi:10.1016/j.renene.2012.08. 064.

[9] M. G. Gebreslassie, G. R. Tabor, M. R. Belmont, Investigation of the performance of a staggered configuration of tidal turbines using CFD, Renewable Energy 80 (2015) 690-698. doi:10.1016/j.renene.2015.03.001. URL http://linkinghub.elsevier.com/retrieve/ $\mathrm{pii} / \mathrm{S} 0960148115001822$

[10] L. E. Myers, A. S. Bahaj, An experimental investigation simulating flow effects in first generation marine current energy converter arrays, Renewable Energy 37 (1) (2012) 28-36. doi:10.1016/j.renene.2011.03.043.

[11] P. Myceka, B. Gaurierb, G. Germainb, G. Pinona, E. Rivoalena, Experimental study of the turbulence intensity effects on marine current turbines behaviour. Part I: One single turbine, Renewable Energy 66 (June) (2014) 729-746.

[12] T. Stallard, R. Collings, T. Feng, J. Whelan, Interactions betweem tidal turbine wakes: Experimental study of a group of 3-bladed rotors, Philosophical Transactions of the Royal Society of London: A 371 (1985) (2013) 1471-2962.

[13] P. Jeffcoate, T. Whittaker, C. Boake, B. Elsaesser, 


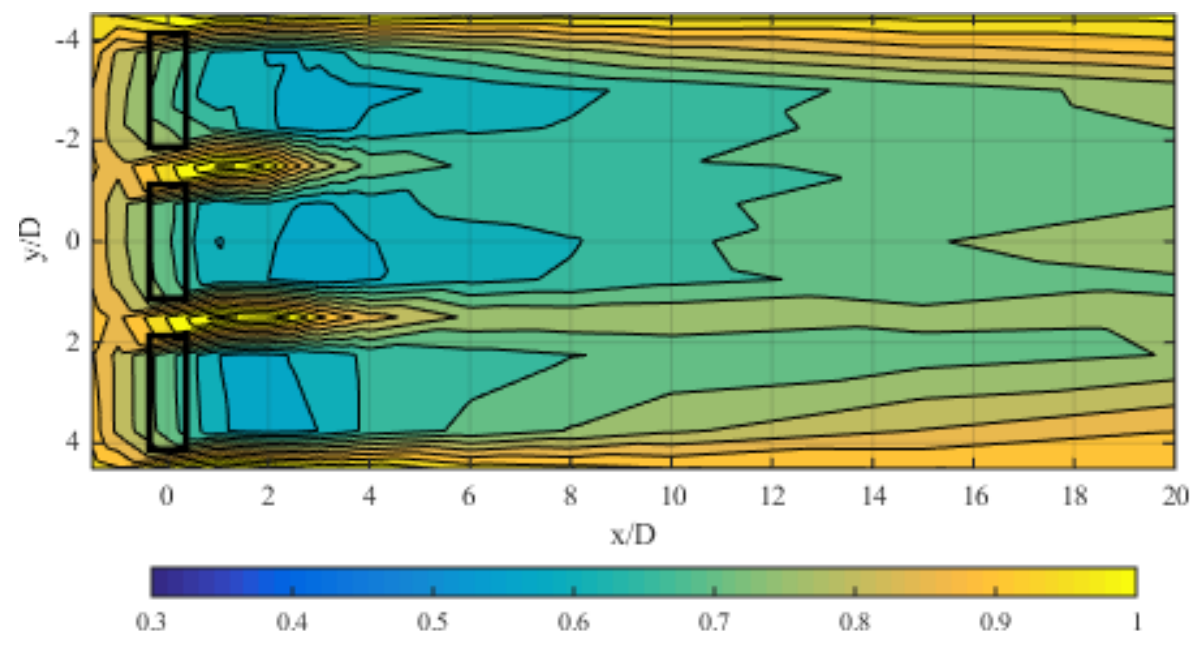

Figure 22: Axial velocity contour given in $\mathrm{m} / \mathrm{s}$ of the three turbine fence array.

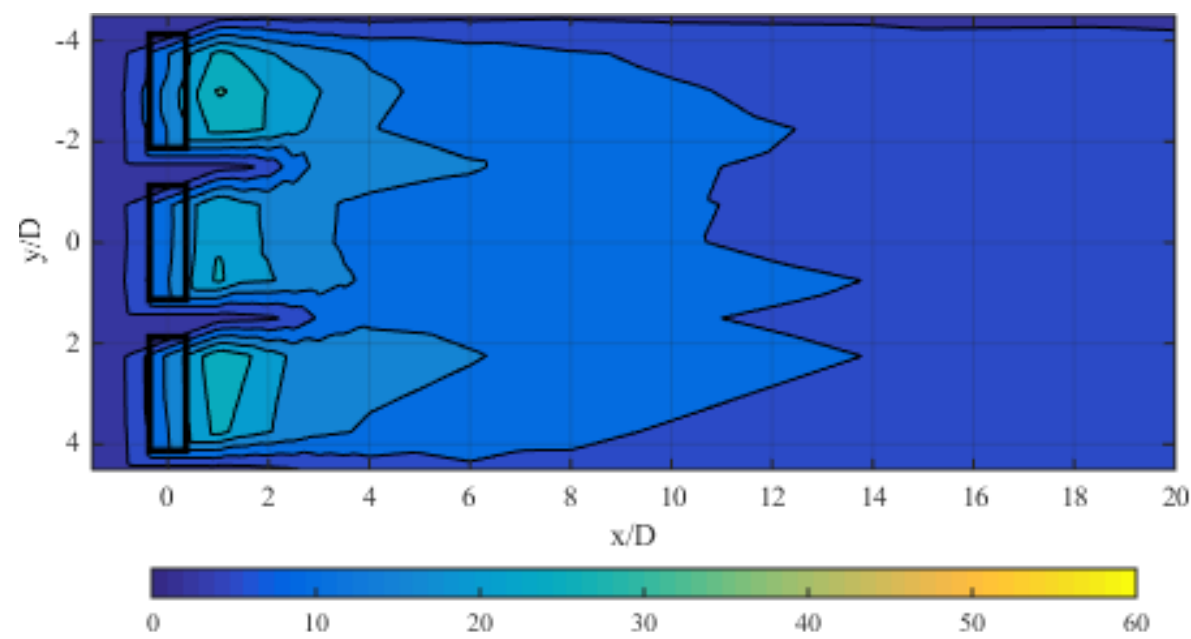

Figure 23: I map of three turbine fence array. Contour map presented in $\%$. 
Field tests of multiple $1 / 10$ scale tidal turbines in steady flows, Renewable Energy 87 (2016) 240-252. doi: $10.1016 /$ j.renene. 2015.10.004.

[14] M. G. Gebreslassie, S. O. Sanchez, G. R. Tabor, M. R Belmont, T. Bruce, G. S. Payne, I. Moon, Experimental and cfd analysis of the wake characteristics of tidal turbines, International Journal of Marine Energy 16 (2016) 209219. doi:10.1016/j.ijome.2016.07.001.

URL http://dx.doi.org/10.1016/j.ijome.2016.07. 001

15] A. P. Janssen, M. R. Belmont, Initial research phase of MRL turbine. Technical report, Technical Report No: MO 562L.

[16] Kinetrol, Model Q-CRD Dashpot (2016).

URL http://www.kinetrol.com/damper/model/ dashpot\{_\}model\{_\}qcrd.html

[17] Crouzet, DC Geared Motor (2016).

URL http://uk.rs-online.com/web/p/ dc-geared-motors/2243596/

[18] Interface, Model T8 ECO Rotary Torque Transducer (2016).

[19] L. A. El-Gabry, D. R. Thurman, P. E. Poinsatte, Procedure for determining turbulence length scales using hotwire anemometry, Tech. rep., NASA/TM2014218403 (2014).

[20] D. Sutherland, S. Ordonez-Sanchez, M. R. Belmont, I. Moon, J. Steynor, Experimental Optimisation of Power for Arrays of Cross Flow Tidal Turbines (2016).

[21] P. Mycek, B. Gaurier, G. Germain, G. Pinon, E. Rivoalen, Numerical and experimental study of the interaction between two marine current turbines, International Journal of Marine Energy 1 (2013) 70-83. arXiv:1310.4921, doi:10.1016/j.ijome.2013.05.007.

[22] P. Bachant, MartinWosnik, Effects of reynolds number on the energy conversion and near-wake dynamics of a high solidity vertical-axis cross-flow turbine, Energies. 